\title{
Oxaliplatin Plus S1 for Treating Sorafenib-Refractory Recurrent Hepatocellular Carcinoma after Liver Transplantation
}

\author{
Jing Sun ${ }^{1}$, Xiaomin Cai ${ }^{1}$, Wei $\mathrm{Li}^{1}$, Meiling Zhang ${ }^{1}$, Jing He ${ }^{1}$, Qian Wang ${ }^{1}$ and Kaihua $\mathrm{Lu}^{1^{*}}$ \\ ${ }^{1}$ Department of Medical Oncology, the First Affiliated Hospital of Nanjing Medical University, Nanjing, Jiangsu, China
}

*Corresponding author: Kaihua Lu, Department of Medical Oncology, the First Affiliated Hospital of Nanjing Medical University, Nanjing, Jiangsu, China, Tel: +862568136714; E-mail: lukaihua@njmu.edu.cn

Received date: May 6, 2017; Accepted date: June 8, 2017; Published date: June 13, 2017

Copyright: ( 2017 Sun J, et al. This is an open-access article distributed under the terms of the Creative Commons Attribution License, which permits unrestricted use, distribution, and reproduction in any medium, provided the original author and source are credited.

\begin{abstract}
Recurrent hepatocellular carcinoma (HCC) following liver transplantation (LT) correlates with a poor prognosis due to the lack of effective therapeutic approaches after sorafenib (SORA) or everolimus (EVL) resistance. We assessed the efficacy of SOX (S-1 and oxaplatin) after failed SORA for HCC recurrence after LT based on the results of next generation sequences (NGS). A patient with HCC recurrence after LT was treated with SORA until severe side effect. And then NGS was performed on the primary and metastatic tumor tissues. Investigations on molecular aberrations in cellular pathways and SNPs of drug-related genes were applied. The result of the first NGS shows the benefit from platinum drugs and no abnormalities in PI3K/AKT/mTOR pathway. He received SOX regimen for 4 cycles with progression-free survival (PFS) as long as 4.3 months. NGS of lung and liver metastasis tissues after disease progression still did not match molecular target of everolimus, which he switched to for experimental therapy. He died of failure treatment with 17.3 months overall survival. SOX regimen may benefit recurrent $\mathrm{HCC}$ after LT. NGS may provide personalized clinical care for HCC patients.
\end{abstract}

Keywords: Hepatocellular carcinoma; SOX; Liver transplantation; Sorafenib

Abbreviations AFP: Alpha-fetoprotein; CT: Computed Tomography; HCC: Hepatocellular Carcinoma; OLT: Orthotopic Liver Transplantation; SORA: Sorafinib; EVL: Everolimus; NGS: Next Generation Sequencing; mTOR: Mammalian Target of Rapamycin; PET-CT: Positron Emission Tomography-Computed Tomography; TACE: Transcatheter Arterial Chemoembolization; SNPs: Single Nucleotide Polymorphisms; PI3K: Phosphatidylinositol 3 Kinase.

\section{Introduction}

Recurrence of hepatocellular carcinoma (HCC) occurs in approximately $8-20 \%$ of patients after liver transplantation (LT) leading to poor long-time survival [1,2]. Compared to various therapeutic approaches used before recurrence, including local treatment, hepatic resection and chemotherapy, no standard posttransplant adjuvant chemotherapy has been established. Sorafinib (SORA) and everolimus (EVL) have already been investigated for application in recurrence HCC after LT [3-6] based on roles of B-Raf and mammalian target of rapamycin (mTOR)/protein kinase B (AKT) pathways in the pathogenesis of HCC $[7,8]$. Targeted next generation sequencing (NGS) of tumor samples can provide molecular abnormalities of different pathways for prediction of treatment outcomes. Although FOLFOX4 (infusional fluorouracil, leucovorin, and oxaplatin) administration has been demonstrated to confer benefit to HCC patients [7], few cases have been reported about its apply in recurrence $\mathrm{HCC}$ after LT.

\section{Case Presentation}

A 44-year-old man was admitted to our hospital due to a $6.9 \times 6.5$ $\mathrm{cm}$ hypoechoic mass in his right hepatic lobe detected by a routine ultra-sound. Pre-transplant serum alpha fetoprotein concentration (AFP) was $14.78 \mathrm{ng} / \mathrm{mL}$ reference range: $<20 \mathrm{ng} / \mathrm{mL}$. He has a history of hepatitis B (HBsAg, $\mathrm{HBeAg}$, anti-HBc positive) for 20 years without receiving any treatment. Positron Emission Tomography - computed tomography (PET-CT) scan demonstrated a mass as $8.6 \times 6.1 \mathrm{~cm}$ in the dome near the junction of the left and right lobe of liver by using SUVmax 6.0. No preoperative biopsy of the liver was performed, with diagnosis as HCC according to medical history and radiologic criteria [9]. While waiting 3 months for the transplantation, the patient underwent one session of trans-arterial chemoembolization (TACE) for stabilizing the disease. Afterward, oral SORA was started at $400 \mathrm{mg}$ twice a day. The patient strongly desired to undergo liver transplantation and underwent orthotopic liver transplantation (OLT) in August 2013. Histopathologic examination revealed poorly differentiated HCC around $9 \times 7 \times 7 \mathrm{~cm}$, grade $3 / 4$ without lymphovascular invasion. Hepatitis B immunoglobulin (HBIG) and entecavir were taken for HBV treatment and HBV DNA subsequently turned negative after that [10]. Rapamycin-based post-transplant immunoprophylaxis without steroids was subsequently initiated after transplantation.

In December 2013, 4 months after LT, HCC recurred as multiple metastases in both lungs that were detected by enhanced computed tomography (CT) and Emission Computed Tomography (ECT) showed multiple bone metastasis. His AFP was elevated to $23.1 \mathrm{ng} / \mathrm{mL}$. Meanwhile, he suffered from a weigh loss of 5Ibs in the past 2 months. SORA was again initiated at the dose of $400 \mathrm{mg}$ twice a day. Zoledronic acid was administered intravenously at a dose of $4 \mathrm{mg}$ every 3 weeks for osteoclast inhibition. AFP rapidly increased up to $47.9 \mathrm{ng} / \mathrm{mL}$ and the patient refused to continue the treatment due to severe diarrhea.

Targeted next generation sequencing (NGS) performed on a tumor sample from the operation detected single nucleotide polymorphisms (SNPs) on rs11615 of ERCC1, rs1801159 of DPYD, indicating probable benefit from platinum drugs and severe side effects from 5 -fluorouracil 
Citation: Sun J, Cai X, Li W, Zhang M, He J, et al. (2017) Oxaliplatin Plus S1 for Treating Sorafenib-Refractory Recurrent Hepatocellular Carcinoma after Liver Transplantation. J Carcinog Mutagen 8: 296. doi:10.4172/2157-2518.1000296

Page 2 of 4

and capecitabine. Meanwhile, SORA and EVL-related pathway genes are all wild-type. He received SOX regimen (Oxaplatin $130 \mathrm{mg} / \mathrm{m}^{2}$ $\mathrm{d} 1+\mathrm{S}-1 \quad 60 \mathrm{mg} \mathrm{d} 1-14,21 \mathrm{~d} / \mathrm{cycle}) \quad(\mathrm{S}-1$, an oral fluorinated pyridimidine, Taiho Pharmaceutical Co Ltd, Tokyo, Japan) from January 2014. A chest CT scan after two cycles of chemotherapy in April 2014 showed a response of about $60 \%$ according to RECIST criteria modifications (Figures $1 \mathrm{~A}-\mathrm{F}$ and $2 \mathrm{~A}-\mathrm{F}$ ).

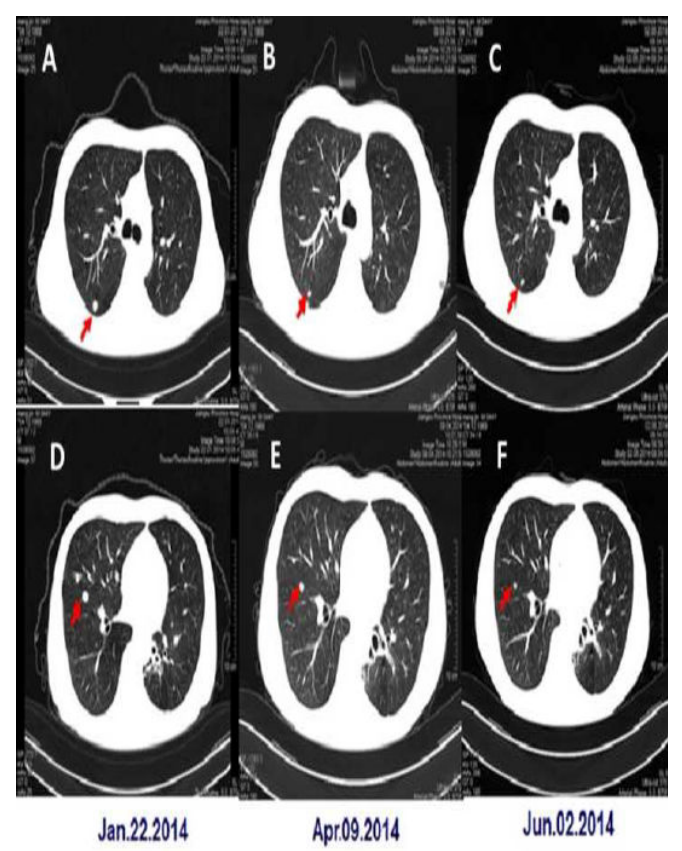

Figure 1: Abdominal and pelvic CT images.

Moreover, the patient tolerated SOX regimen very well, without occurring neutropenia and hematologic toxicity. His AFP level decreased to $7.6 \mathrm{ng} / \mathrm{mL}$. A follow-up CT scan after four cycles also revealed marked shrinkage in lung nodules (Figures $1 \mathrm{~A}-\mathrm{F}$ and $2 \mathrm{~A}-\mathrm{F}$ ). However, abdominal and pelvic CT images at that time showed recurrence on the $\mathrm{S} 6$ segment in the right lobe of the liver. In addition, his AFP was again elevated to $24.9 \mathrm{ng} / \mathrm{mL}$. NGS analysis on two tumor samples from the lung and the liver biopsy after progression showed all wild-type phenotypes of SORA and EVB-related pathway genes as reported previously. Subsequently he received EVL therapy with $10 \mathrm{mg}$ orally daily from July 2014, which did not match any detected molecular abnormality. A CT scan performed after 1.5 months showed progression not only in his lung but also in liver metastases.

Severe (Grade 3) hepatic dysfunction and mild (Grade 1) fatigue developed according to common terminology criteria for adverse events (CTCAE) v 3.0. The patient did not receive other experimental therapy due to his worsening performance status. He was managed with supportive care and died of progressive disease in November 2014. The overall survival was 17.3 months.

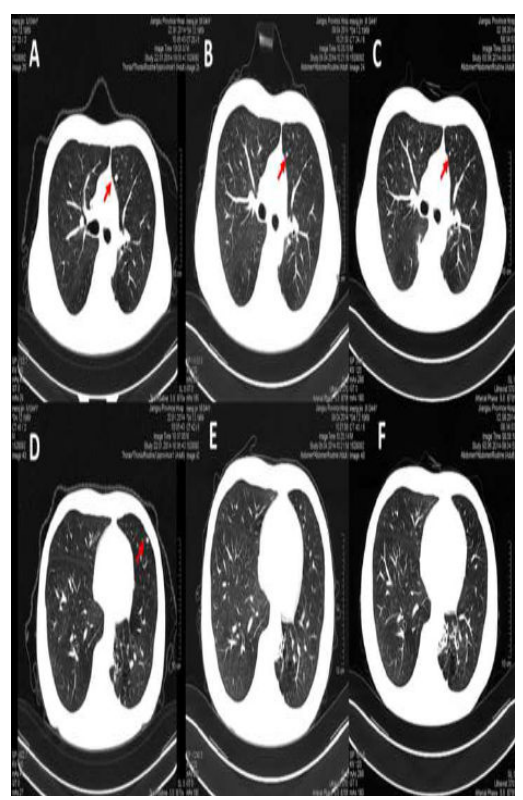

Figure 2: Abdominal and pelvic CT images.

\section{Discussion}

This is a case of illustrating SOX regimen as alternative treatment for metastatic HCC recurrence after LT. The case is especial for SORA and EVL-resistance HCC. Although SORA and EVL are generally recommended sequentially or co-instantaneously, there is still lack of optimal treatment modality $[2-4,6]$. No alternative drug or treatment had shown an obvious survival benefit in HCC with SORA or EVLresistant after transplant [11]. Four months after liver transplantation, the patient developed recurrent HCC in both lungs. The administration of SOX regimen induced partial release (PR) in the lung metastases after refractory to SORA and PFS was 4.3 months.

In general, chemotherapy is not recommended regularly in HCC for its resistance [12,13]. However, the EACH study, first large, international, multicenter phase III study of systemic chemotherapy in 2013, reported that FOLFOX4 (infusional fluorouracil, leucovorin and oxaliplatin) regimen can confer benefit to Asian patients, although EACH study patients were more heavily pretreated, more HBV infection, fewer Child-Pugh A compared with SHARP study [14]. Moreover, in pivotal phase III trials, the survival benefits of SORA were less prominent in Asian than in Western patients [15,16], possibly due to the hereditary difference. For our patient, TACE and SORA were performed together to control the tumor size in the interval. In addition, SORA probably proved ineffective for controlling lung metastasis. So the efficiency of SORA is difficult to be judged. The reason may be that the patient is primary refractory to SORA, but not acquired resistance. NGS performed on the primary tumor sample also showed no mutations on SORA-related pathway genes, indicating less possibility for availability.

This is also a case of an individualized molecular approach aimed to identify aberrant activation of the principal signal routes deregulated in HCC in a patient with recurrent tumors after liver transplantation. 
The genomic landscape of HCC is unclear until now. A personalized approach aimed to treat recurrent hepatocarcinoma is possible through analysis of tumoral molecular pathways. Partial success of the selected everolimus supports the vital role of mTOR signaling and highlights the importance of reliable biomarkers to route the best molecular-based therapeutic options in HCC. To detected molecular aberrations which led to putative activation of the PI3K/AKT/mTOR pathway, Wnt pathway, MAPK pathway, aberrant DNA repair mechanisms, cell cycle control and apoptosis, the NGS method was applied on his tumor samples using the Illumina HiSeq 4000 platform.

NGS data were obtained for 3 tumor tissues (1 primary lesion, 1 lung metastatic foci and 1 recurrence of liver transplantation) and 1 normal tissue as negative control from the patient with metastatic HCC (hepatitis B-related HCC). As a result, several novel mutations (FGFR3 E47A, CDKN2B A87S, POLE I238F, MSH6 X1231delinsX) only in recurrence of liver transplantation were detected by NGS. And their clinical significances are unknown. These mutations may contribute to the occurrence and development of tumors as drivers in this patient.

According to RECIST assessments after each line therapy, this patient had different responses to SOX chemotherapy in lung metastatic foci (partial response) and the recurrence of liver transplantation (progressive disease) respectively. NGS performed on the tumor samples from lung metastatic foci and the liver transplant after diagnosis revealed TP53 R249S mutation and BLM G1049A mutation in common. It suggested the two tumors had the same source which traced to the primary lesion. The mutant frequency of TP53 was $31 \%$ in cases of HCC as reports [17]. TP53 R249S is located within the DNA-binding domain of the p53 protein, which is thought to result in loss of function. BLM G1049A mutation hasn't been reported in HCC in the COSMIC database. However, there is heterogeneity within most tumors. HCC is also included. FGFR3 E47A mutation, CDKN2B A87S mutation, POLE I238F mutation and MSH6 X1231delinsX mutation were first diagnosed in the recurrence of liver transplantation. The molecular status of these tumor cells showed that they would be from other sources or new generated tumors. These novel mutations may lead to different responses to SOX chemotherapy.

mTOR signalling is aberrantly activated in about $50 \%$ of human HCCs. And different molecular alterations underline mTOR deregulation (EGFR up-regulation, RAS/MAPK, PI3K/AKT upregulation, PTEN down-regulation, RICTOR copy number gain) [18]. In the presented case, three tumor tissues had no molecular aberration with putative activation of the PI3K/AKT/mTOR pathway, Therefore, the patient didn't obtain therapeutic benefit from Everolimus by RECIST assessment after receiving mTOR inhibitor therapy.

A sub-analysis showed that the worst group of patients (recurrence $<1$ year, bone metastasis, no surgical treatment after recurrence) survived a significantly shorter period, with overall survival (OS) and 1 - year survival rate for approximately 6 months and $10 \%$, respectively [19]. Although this patient was in the worst group mentioned above, he had a relatively favorable outcome after treatment with SOX. Further investigation will be required to apply this therapy to recurrent HCC after LT for safety and efficacy.

\section{Acknowledgement}

This work is supported by National Natural Science Foundation of China (No 81302109) to J Sun.

\section{References}

1. Roayaie S, Schwartz JD, Sung MW (2004) Recurrence of hepatocellular carcinoma after liver transplant: patterns and prognosis. Liver Transpl 10: 534.

2. De Simone P, Crocetti L, Pezzati D, Bargellini I (2014) Efficacy and safety of combination therapy with everolimus and sorafenib for recurrence of hepatocellular carcinoma after liver tansplantation. Transplant Proc 46: 241-244.

3. Bhoori S, Toffanin S, Sposito C, Germini A (2010) Personalized molecular targeted therapy in advanced, recurrent hepatocellular carcinoma after liver transplantation: a proof of principle. J Hepatol 52: 771-775.

4. Waghrav A, Balci B, EI-Gazzaz G, Kim R (2013) Safety and efficacy of sorafenib for the treatment of recurrent hepatocellular carcinoma after liver transplantation. Clin Transplant 2: 555-561.

5. Sposito C, Mariani L, Germini A, Flores Reyes M, Bongini M, et al. (2013) Comparative efficacy of sorafenib versus best supportive care in recurrence hepatocellular carcinoma after liver transplantation: a casecontrol study. J Hepatol 59: 59-66.

6. Gomez-Martin C, Bustamante J, Castroagudin JF, Salcedo M, Garralda E, et al. (2012) Efficacy and safety of sorafenib in combination with mammalian target of rapamycin inhibitors for recurrent hepatocellular carcinoma after liver transplantation. Liver Transpl 18: 45-52.

7. Bhoori S, Toffanin S, Sposito C, Germini A (2010) Personalized molecular targeted therapy in advanced, recurrent hepatocellular carcinoma after liver transplantation: a proof of principle. J Hepatol 52: 771-775

8. De Simone P, Crocetti L, Pezzati D, Bargellini I (2014) Efficacy and safety of combination with everolimus and sorafenib for recurrence of hepatocellular carcinoma after liver transplantation. Transplant Proc 46: 241-244

9. Bruix J, Sherman M (2005) Management of hepatocellular carcinoma. Hepatology 42: 1208-1236.

10. Campsen J, Zimmerman M, Trotter J, Hong J (2013) Liver transplantation for hepatitis B liver disease and concomitant hepatocellular carcinoma in the United States with heptatitis B immunoglobulin and nucleoside/ nucleotide analogues. Liver Transpl 19: 1020-1029.

11. De'Angelis N, Landi F, Carra MC, Azoulay D (2015) Managements of recurrent hepatocellular carcinoma after liver transplantation: A systematic review. World J Gastroenterol 21: 11185-11198.

12. Park JG, Lee SK, Hong IG (1994) MDR1 gene expression: Its effect on drug resistance to doxorubicin in human hepatocellular carcinoma cell lines. J Natl Cancer Inst 86: 700-705.

13. Park JG (2000) Expression of P-glycoprotein in hepatocellular carcinoma. A determination of chemotherapy response. Am J Clin Pathol 113: 355-363

14. Qin S, Bai Y, Lim HY, Thongprasert S (2013) Randomized, multicenter open-label study of oxaliplatin plus flurrouracil/leucovorin versus doxorubicin as palliative chemotherapy in patients with advanced hepatocellular carcinoma from Asia. J Clin Oncol 31: 3501-358.

15. Cheng AL, Kang YK, Chen Z (2009) Efficacy and safety of sorafenib in patients in the Asia-Pacific region with advanced hepatocellular carcinoma; A phase III randomized, double-blind, placebo-controlled trial. Lancet Oncol 10: 25-34.

16. Llovet JM, Ricci S, Mazzaferro V (2008) Sorafenib in advanced hepatocellular carcinoma. N Engl J Med 359: 378-390

17. Forbes SA, Bindal N, Bamford S, Cole C, Kok CY, et al. (2011) COSMIC: mining complete cancer genomes in the Catalogue of Somatic Mutations in Cancer. Nucleic Acids Res 39: D945-950.

18. Villanueva A, Chiang DY, Newell P, Peix J, Thung S, et al. (2008) Pivotal role of mTOR signaling in hepatocellular carcinoma. Gastroenterology 135: 1972-1983.

19. Schwartz M, Roayaie S, Llovet J (2005) How should patients with hepatocellular carcinoma recurrence after liver transplantation be treated. J Hepatol 43(4): 584-589. 